\title{
Predicting Rare Classes: Comparing Two-Phase Rule Induction to Cost-Sensitive Boosting
}

\author{
Mahesh V. Joshi ${ }^{1}$, Ramesh C. Agarwal ${ }^{2}$, and Vipin Kumar ${ }^{3}$ \\ 1 IBM T. J. Watson Research Center \\ P.O. Box 704, Yorktown Heights, NY 10598, USA \\ joshim@us.ibm.com \\ 2 IBM Almaden Research Center \\ 650 Harry Road, San Jose, CA 95120, USA \\ ragarwal@us.ibm.com \\ 3 Dept. of Computer Science and AHPCRC, University of Minnesota \\ 1100 S. Washington Ave, Minneapolis, MN 55415, USA \\ kumar@cs.umn.edu
}

\begin{abstract}
Learning good classifier models of rare events is a challenging task. On such problems, the recently proposed two-phase rule induction algorithm, PNrule, outperforms other non-meta methods of rule induction. Boosting is a strong meta-classifier approach, and has been shown to be adaptable to skewed class distributions. PNrule's key feature is to identify the relevant false positives and to collectively remove them. In this paper, we qualitatively argue that this ability is not guaranteed by the boosting methodology. We simulate learning scenarios of varying difficulty to demonstrate that this fundamental qualitative difference in the two mechanisms results in existence of many scenarios in which PNrule achieves comparable or significantly better performance than AdaCost, a strong cost-sensitive boosting algorithm. Even a comparable performance by PNrule is desirable because it yields a more easily interpretable model over an ensemble of models generated by boosting. We also show similar supporting results on real-world and benchmark datasets.
\end{abstract}

\section{Introduction and Motivation}

In many domains such as fraud detection, network intrusion detection, text categorization, and web mining, it is becoming critical to be able to learn predictive, high precision models for some important events that occur very rarely. In most of these domains, the data is available in a labeled form enabling use of classification methods. We focus on the binary classification problem in this paper. The goal is to build a model for distinguishing one rare class from the rest.

Some work has started to emerge to solve this problem $[1,2,3]$. In the context of rare classes, we take a stand similar to the Information Retrieval community, that a meaningful evaluation metric should reflect a balance between the recall and precision of the given rare class ${ }^{1}$. From this recall-precision perspective,

\footnotetext{
${ }^{1}$ If a classifier detects $m$ examples to be of class $\mathrm{C}$, out of which $l$ indeed belong to $\mathrm{C}$, then its precision $(P)$ for class $\mathrm{C}$ is $l / m$. If $\mathrm{C}$ has total of $n$ examples in the set, then

T. Elomaa et al. (Eds.): PKDD, LNAI 2431, pp. 237-249, 2002.

(C) Springer-Verlag Berlin Heidelberg 2002
} 
a recently proposed two-phase rule-induction algorithm PNrule, was shown to outperform single-phase methods such as RIPPER and C4.5rules [3], because of its way of decoupling the recall and precision objectives.

In the past few years, boosting has emerged as a competitive technique. Various boosting algorithms have been proposed in the literature $[5,6,7,8]$. From the traditional goal of accuracy, theoretical analysis [5] has been conducted to show that boosting can always improve accuracy as the iterations progress, as long as its base learner satisfies the weak learnability criteria of the PAC theory ${ }^{2}$. However, no such theoretical analysis has been done to see if boosting can always improve the recall-precision based performance that we desire for the rare classes. Recently, we compared the effect of weight update mechanisms on various boosting algorithms [9] for the rare class prediction problem. AdaCost [8], a cost-sensitive algorithm emerged as a strong algorithm as compared to all others because of its ability to implicitly emphasize both recall and precision.

Boosting results in an ensemble of models, hence we refer to it as a metatechnique as opposed to the non-meta techniques that generate a single model. In this paper, we take the case of the two strong algorithms from each category; viz. PNrule and AdaCost, and make an attempt to see if one key feature of PNrule is implicitly present in boosting. We present detailed qualitative analysis to argue that boosting does not guarantee an effect similar to this feature over its iterations. This fundamental difference in the two algorithms leads us to expect existence of many scenarios in which PNrule will be comparable to or significantly better than boosting from the recall-precision perspective. Even if PNrule performance is comparable, it is preferable in many domains such as document categorization or network intrusion detection where easy interpretability of the model is highly desirable.

We now briefly illustrate the feature of PNrule via an example. Let us assume that we are building a model for a network intrusion attack of type remote-tolocal ( $\mathrm{r} 2 \mathrm{l})$. It can be distinguished via rules based on attributes such as protocol_type (tcp, udp, etc), number_of_logins, and service_type (ftp, http, etc). But, some such rules, e.g. service_type $=$ http, may also capture many false positives of the denial-of-service (dos) attack. In order to increase precision of r2l's model, these false positives of dos must be removed by learning rules such as duration_of_connection $<2$ seconds $\rightarrow$ dos or bytes_transferred $<200 \rightarrow$ dos. Now, these rules can be learned in two ways: implicitly or explicitly. The implicit way is to add to each $\mathrm{r} 2 \mathrm{l}$ rule the conjunctive conditions that predict absence of dos. The single-phase algorithms (e.g. RIPPER [10]) do an implicit learning. An explicit approach gathers all the examples covered by at least one of the r2l rules, and then explicitly learns rules for the presence of dos from this collection. PNrule's learning method is precisely this explicit approach. For implicit methods to perform well, the rules for the absence of dos should be learned in their

the classifier's recall $(R)$ for $\mathrm{C}$ is $l / n$. Balance between $R$ and $P$ can be measured in various ways such as $F$-measure [4], the $R=P$ point, etc.

${ }^{2}$ A weak learner is an algorithm that, given $\epsilon \leq 1 / 2-\gamma(\gamma>0)$ and $\delta>0$, can achieve an error rate of $\epsilon$, with a probability of at least $(1-\delta)$. 
totality for every $\mathrm{r} 2 \mathrm{l}$ rule. This may not be always possible. One such situation is when dos has many disjoint conditions for its presence, and its examples are split across many rules of $\mathrm{r} 2$, to an extent that dos rules or absence thereof may not be completely learned via the piecemeal approach taken by implicit methodology. The explicit approach has an advantage in such situations by being able to collect the false positives of dos together and learn stronger rules to exclude them.

In this paper, our key contribution is the detailed qualitative analysis to argue that boosting does not impart an explicit learning capability to its base algorithm that learns using the implicit approach. Boosting can achieve higher recall via its intelligent yet incremental weight updating mechanism, which translates into removing the relevant false positives also in a piecemeal fashion. Thus, it may not imitate the effect of collecting the false positives to learn their complete or correct description, and hence fail to achieve a good balance between recall and precision. In the rest of the paper, we elaborate this reasoning further and support our arguments empirically on a wide range of synthetic and real-world scenarios.

\section{Overview of Algorithms}

We first briefly describe the two key algorithms being compared in this paper.

\section{Boosting:}

All the boosting algorithms (AdaBoost [5], SLIPPER [6], AdaCost [8], CSB1, CSB2 [7], and RareBoost [9]) work in iterations, each time learning a classifier model via a weak learner on a different weighted distribution of training records. After each iteration, weights on all the examples are updated in a manner that forces the weak classifier to focus more on the incorrectly classified examples in the next iteration. In the end, prediction of an example's class is made using the classifiers learned in all the iterations via a weighted voting process. For the rare class problem, a crucial factor that distinguishes different algorithms is the differences in the weight update factors [9]. For a cost-sensitive boosting algorithm AdaCost, here are the weight update equations from iteration $t$ to $(t+1)$ for the four types of examples defined with respect to the rare class (TP: true positive, FP: false positive, TN: true negative, and $\mathrm{FN}$ : false negative) [9]: $T P_{t+1} \rightarrow T P_{t} / \gamma, T N_{t+1} \rightarrow T N_{t} / \gamma, F P_{t+1} \rightarrow F P_{t} * \gamma^{(f+1) / f}, F N_{t+1} \rightarrow F N_{t} * \gamma^{2}$, where $f \geq 1$ is the cost-factor given to the misclassification of the rare class (false negatives) $; \gamma=e^{0.5 \alpha_{t}}$; and $\alpha_{t}$ is the strength assigned to the vote of $t^{\text {th }}$ classifier in the voting process. Although we will present our arguments with AdaCost in mind, they are also applicable to other boosting algorithms.

\section{PNrule:}

Given a training data-set and the target class, the PNrule algorithm [3] learns a binary two-phase model for the target class. PNrule framework was introduced first in [2], where it was also successfully extended to the multi-class problems 
of the network intrusion detection domain. It was later analyzed in detail for the rare class problems in [3] to show its strength over single-phase algorithms such as RIPPER [10] and C4.5rules [11]. PNrule learns a model of disjunctive normal form (DNF) consisting of two kinds of rules: P-rules predicting presence of the target rare class $\mathrm{C}$ and N-rules predicting absence of $\mathrm{C}$. The first phase of learning (P-phase) learns P-rules in a sequential covering manner The goal is to achieve high recall for $\mathrm{C}$ by inducing high support rules. Accuracy is traded off in favor of support in the later iterations, in order to minimize exposure to the small disjuncts problem [3]. This leads to a less precise model. Then, the true and false positives covered by P-rules are collected together and the second phase (N-phase) learns N-rules to remove the false positives collectively. The goal is to improve overall precision, while keeping recall at an acceptable level. $\mathrm{N}$-phase also follows sequential covering ${ }^{3}$.

The addition of rules in P-phase and rule growth in the N-phase are driven by two lower recall limit parameters, $r p$ and $r n$, resp. Usually, these can be tuned for achieving better performance. Due to lack of space, we refer the reader to $[2,3]$ for details of these and other parameters.

\section{Boosting vs. PNrule: What Lacks in Boosting?}

In this section, we present qualitative arguments to show that one crucial feature of PNrule is lacking in the boosting mechanism.

PNrule collects all the examples covered by the union of P-rules, and learns rules to remove false positives from this collection to improve precision. This feature is pictorially illustrated in Figure 1, which shows a snapshot of the process of learning a model to distinguish rare class $\mathrm{C}$ from the other class NC. First phase learns the P-rules $P_{i}$. Second phase then learns N-rules on the union of $P_{i}$-covered examples, to regain precision. This explicit learning approach of PNrule allows it to learn a strong N-rule such as $N_{0}$. Now, a single-phase method such as RIPPER [10] has to learn $N_{0}$ implicitly by learning the absence of each of its pieces intersecting with each $P_{i}$. It can perform as well as PNrule only if there are sufficient number of examples available to learn a complete description for the absence of each of these pieces. As shown in [3], there exist many situations where this condition is difficult to meet, especially when the complete description of absence consists of large number of conjunctive conditions. There are other reasons also [3], such as reducing the small disjuncts problem, that motivate PNrule's preference for some less accurate $P_{i}$ rules, which in turn requires learning of a rule such as $N_{0}$. Thus, in many situations, it is crucial to have an ability to achieve an effect similar to that of learning $N_{0}$ from the collection of multiple P-rules.

\footnotetext{
${ }^{3}$ PNrule's philosophy is similar to the concept of counterfactuals [12] or ripple-downrules [13], from first order logic learning and knowledge based systems, respectively. To our knowledge, there is no evidence of their applicability and scalability to large real-world problems of propositional learning that PNrule addresses.
} 


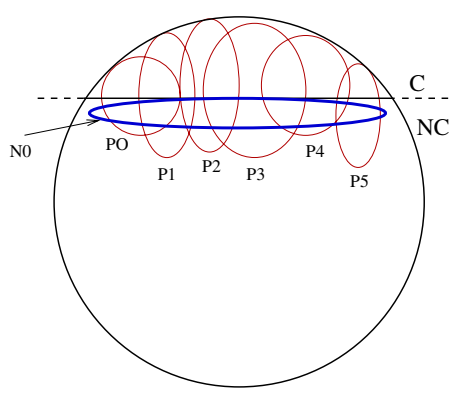

Fig. 1. PNrule's ability to collectively remove false positives with strong N-rules

Now, we try to qualitatively argue that the mechanism of boosting does not have the ability to effectively capture a rule such as $N_{0}$, given that its base learner follows an implicit learning approach. In any given iteration, boosting instructs the weak classifier to operate on a weighted distribution of the training examples. The incremental process of weight update is illustrated in Figure 2. The figure shows first two iterations of a boosting algorithm. Part I shows the decision boundary of classifier $\mathrm{C} 1$ learned in the first iteration. Within this boundary, every example is predicted as $\mathrm{C}$ and outside of it, all are predicted as $\mathrm{NC}$.

At the end of this iteration, weights of the true positive (TP) examples of region $\mathrm{J}$ and the true negative $(\mathrm{TN})$ examples of region $\mathrm{L}$ are reduced, while weights on the false negative (FN) examples of region $\mathrm{K}$ and false positive (FP) examples of region $\mathrm{M}$ are increased. Now, the weak classifier geared towards learning a model for $\mathrm{C}$ tries to focus more on capturing the $\mathrm{C}$ examples with high weight and avoid capturing the high weight $\mathrm{NC}$ examples. In the process, it may capture some NC examples whose weight has become small. Similarly, a weak classifier geared for learning NC's model, tries to capture more of NC's examples with higher weights and less of C's examples bearing higher weights. The net effect is to force the boundary of the next classifier to shift to C2 (part II) that covers more of region $\mathrm{K}$, less of regions $\mathrm{J}$ and $\mathrm{M}$ and possibly some of region $\mathrm{L}$.

Now, after this iteration all the examples get divided into eight different types as shown by eight different regions $(\mathrm{P}-\mathrm{W})$. The weights, calculated using the AdaCost formulae of Section 2, and the ensemble-based predictions of the examples in each of these regions are shown at the second level of each tree in part III of the figure. The relative strengths of classifiers $\mathrm{C} 1$ and $\mathrm{C} 2$ will determine whether examples in regions $\mathrm{Q}, \mathrm{R}, \mathrm{U}$, and $\mathrm{V}$ are predicted as $\mathrm{C}$ or NC. For example, if $\alpha_{2}>\alpha_{1}$, then examples in $\mathrm{U}$ are predicted as belonging to $\mathrm{C}$. Also, unless $\alpha_{1}=\alpha_{2}$, examples in $\mathrm{U}$ cannot have the same prediction as examples in V. Similarly for Q and R. In fact, the final prediction of any example after a given number of iterations will be determined by the total strength of classifiers predicting it as positive versus total strength of the classifiers predicting it as negative. The effect of $\mathrm{U}$ and $\mathrm{V}$ being captured by one N-rule of the PNrule 


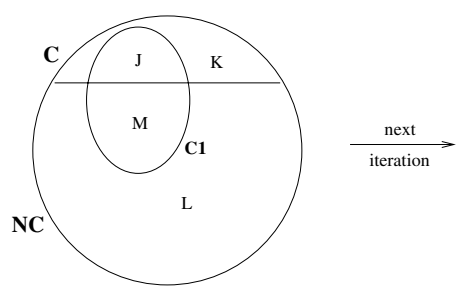

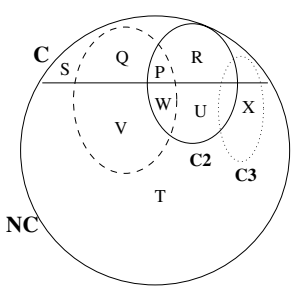

(II)

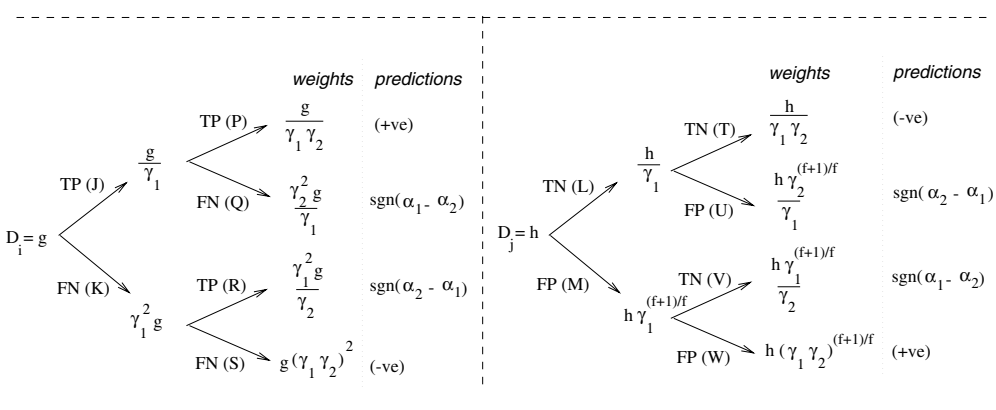

(III)

Fig. 2. Journey of the weight of a given example in boosting iterations. I. After first iteration. II. After second iteration. III. How weights change for each region in I and II, and how predictions are made. $D_{i}$ is weight of example $i$ of class C. $D_{j}$ is weight of example $j$ of class NC

algorithm, is equivalent to a simultaneous prediction of both $\mathrm{U}$ and $\mathrm{V}$ as true negatives by the ensemble of classifiers. We can clearly see that boosting cannot do this with the ensemble of $\mathrm{C} 1$ and $\mathrm{C} 2$. However, one can argue that going one more iteration where the classifier $\mathrm{C} 3$ covers a region as shown in part II of the figure may solve the problem. Now, the prediction for region $\mathrm{U}$ is $\operatorname{sgn}\left(\alpha_{2}-\alpha_{1}-\alpha_{3}\right)$; for region $\mathrm{V}$, it is $\operatorname{sgn}\left(\alpha_{1}-\alpha_{2}-\alpha_{3}\right)$; and for region $\mathrm{X}$, it is $\operatorname{sgn}\left(\alpha_{3}-\alpha_{1}-\alpha_{2}\right)$. As long as the sum of each pair of $\alpha_{1}, \alpha_{2}$, and $\alpha_{3}$ is greater than the third value, each of the regions $\mathrm{U}, \mathrm{V}$, and $\mathrm{X}$ can be predicted as true negative simultaneously by the 3 -ensemble model, thus achieving an effect similar to learning a N-rule encompassing the three. Although boosting theory [5] suggests how to choose $\alpha$ for each iteration, the boosting method does not have any control over the relationship between the $\alpha$ values across iterations. Thus, the desired relationship between $\alpha_{1}, \alpha_{2}$, and $\alpha_{3}$ values cannot be guaranteed. This lack of guarantee essentially indicates the existence of situations where boosting cannot achieve the effect of collecting many false positives required for learning their stronger description, and hence it can fail to achieve good precision for a reasonable recall level.

Generic expressions of $\alpha_{t}$ values are needed to formally support the above statement. Even for a simpler form of AdaCost, which assumes that each base learner $t$ gives a model $h_{t}\left(x_{j}\right) \rightarrow\{0,1\}$ (ignoring any confidence rating), we 
get $\alpha_{t}=0.5 \ln \left(\left(1+r_{t}\right) /\left(1-r_{t}\right)\right)$, where $r_{t}=0.5 \sum_{i: T P, T N} D_{t}(i)+0.5(1+$ $1 / f) \sum_{i: F P} D_{t}(i)-\sum_{i: F N} D_{t}(i)$. The value $D_{t}(i)$ is the weight of $i^{t h}$ example, which depends on the $\gamma_{t}$ values for all the classifiers learned upto iterations $t-1$. For example, after iteration $\mathrm{T}$, weight of an example of class $\mathrm{NC}$ is:

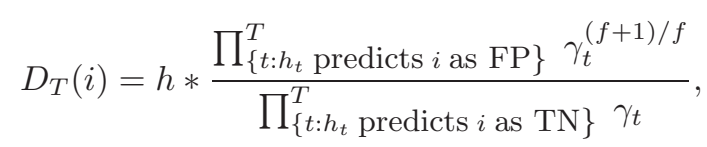

where $h$ is its initial weight. Each $\gamma_{t}$ in turn depends on $\alpha_{t}$ (Section 2). Thus, $D_{t}$ and $\alpha_{t}$ have a complex cyclic relationship, which makes it difficult to perform rigorous formal analysis. This is the reason we resort to the qualitative analysis and arguments.

As the expressions for $r_{t}$ and $\alpha_{t}$ above indicate, if the false positive region (such as $\mathrm{U}, \mathrm{V}$, or $\mathrm{X}$ ) of a base classifier model $h_{t}$ is larger, then $r_{t}$ and $\alpha_{t}$ values for $h_{t}$ will be higher. Thus, the contribution of $h_{t}$ to overall weight will be significant. Moreover, this region will have possibly large number of overlaps with the false positive regions of the other classifiers. So, in the ensemble-based voting process, an example in this region will get high vote as a false positive, thus diminishing its chances of getting correctly predicted. Of course, the weight update mechanism, as illustrated in the part III of Figure 2, will attempt to avoid such situation by increasing the weights of examples in this region, so that fewer and fewer future classifiers cover the region. But, as fewer classifiers cover the region (thus more classifiers treating the examples as TN), Equation 1 indicates that the weights on the examples will either stabilize or start reducing. The relative strengths of the classifiers covering the region will determine the time at which the weight increases sufficiently for the region not to be covered or decreases to an extent that the region is again vulnerable for misclassification. This is a cyclical process, and depending on when an example switches roles among FP and TN, the effect of collective removal of the false positives may be only partially achievable.

In summary, we have qualitatively argued that the incremental nature of weight update mechanism and the ensemble-based voting process are not sufficient to allow boosting to impart an explicit learning effect, similar to that of PNrule, to a base learner that uses an implicit approach.

\section{Results}

We now attempt to empirically support the qualitative arguments of section 3. Two variations of the IREP* [10] algorithm are used as the base learners for boosting. IREP*-1 learns C's model and NC is the default class. IREP*-2 learns a model for $\mathrm{NC}$ also from the whole training set ${ }^{4}$.

We use $F_{1}$-measure [4] as the performance metric, defined as $(2 \mathrm{R} \mathrm{P}) /(\mathrm{R}+\mathrm{P})$, where recall $(\mathrm{R})$ and precision $(\mathrm{P})$ are with respect to $\mathrm{C}: \mathrm{R}=\mathrm{TP} /(\mathrm{TP}+\mathrm{FN})$ and

\footnotetext{
${ }^{4}$ PNrule learns NC's model using only the examples covered by at least P-rule.
} 


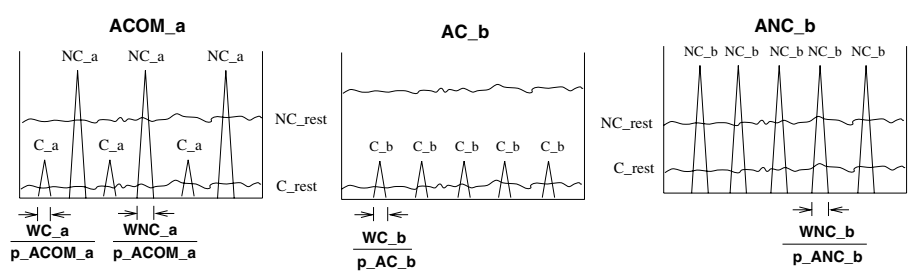

(I)

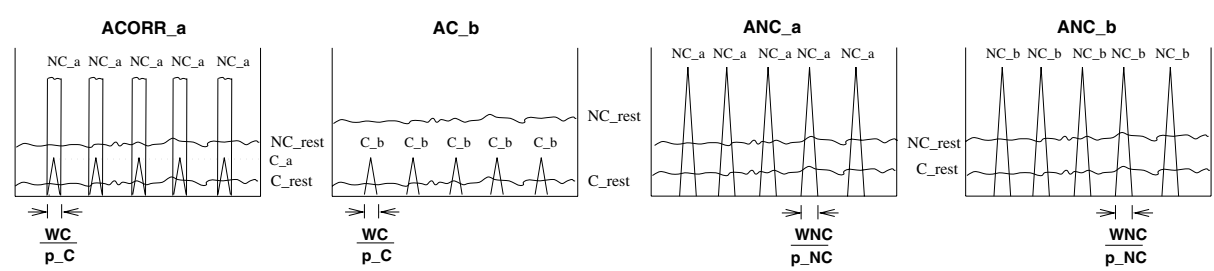

(II)

Fig. 3. Models generating synthetic data. (I): No correlations among attributes. (II): With correlations among attributes of types $A C O R R_{a}$ and $A N C_{a}$ for subclass $N C_{a}$

$\mathrm{P}=\mathrm{TP} /(\mathrm{TP}+\mathrm{FP})$. We use $\mathrm{P}$ instead of a false positive rate of $\mathrm{FP} /(\mathrm{FP}+\mathrm{TN})$ because we are interested in minimizing false positives of $\mathrm{C}$ with respect to the total examples predicted as C. Also other domains such as information retrieval which are concerned with $\mathrm{R}$ and $\mathrm{P}$ values commonly use the $F_{1}$ metric.

For AdaCost, we use various values for $f: 1,2,5,10$. For each $f$ value, we run 50 and 25 iterations with IREP*-1 and IREP*-2, respectively. The parameters $f$ and the number of iterations, are chosen to yield the highest $F_{1}$ value on the validation data (one-third random sample of the training data). For PNrule, the parameters chosen are the rule evaluation function (z-number [2] or information gain [10]), $r p$ (0.7 or 0.95$)$, and $r n$ (0.0 or 0.7$)$. The optimal parameters values are used to report $F_{1}$ values on the test data.

Two different algorithms are compared by first using the p-test ${ }^{5}$ with $95 \%$ confidence level [14] to compare their $\mathrm{R}$ and $\mathrm{P}$ metrics individually. If either metric is better for a classifier and the other metric is comparable or better, then it is a better classifier. If one metric is better and the other worse, then the classifier with higher $F_{1}$ value is better. This method is driven more by the actual $\mathrm{R}$ and $\mathrm{P}$ comparisons rather than a pure $F_{1}$-based comparisons.

\subsection{Results on Synthetic Datasets}

We generate various synthetic datasets by using two different data models described in Figure 3. Each model has different types of attributes. Type is

\footnotetext{
${ }^{5}$ p-test can be thought of as a two-sided t-test around mean.
} 
identified by the nature of the subclass distribution over the attribute. These histogram-based models were first introduced in [3] and were designed to test PNrule's 2-phase nature. The key idea is to split the examples of rare classes into multiple peaks in the distribution and split the number of non-rare class examples into multiple subclasses. We also make each subclass distinguishable by only one attribute or two correlated attributes. For example, $A N C_{b}$ can distinguish $N C_{b}$ subclass of the non-rare class, because of all attributes, $N C_{b}$ 's distribution is non-random only over $A N C_{b}$, and of all subclasses, only $N C_{b}$ is non-randomly distributed over $A N C_{b}$. So, for an implicit (or one-phase) rule induction approach, the descriptions of false positives (i.e. subclasses of $N C$ ) must be learned from the examples that fall in the non-peak regions of the $A N C$ attributes. Instead, PNrule needs to learn the peak regions in the second phase where it explicitly learns false positive descriptions on the collection of examples covered by at least one P-rule. As we stated in the introduction, the goal is to see if boosting mechanism imparts an explicit learning capability to an implicit base learner. These datasets will help us do precisely that.

For each model, there are several parameters (some defined in Figure 3) such as the number of subclasses of each type, the number of peaks and the peak width for a given type of attribute (e.g. for type $A C_{b}$ there are p_AC_b peaks with total width of WC_b), and the proportion of $\mathrm{C}$ vs. NC (the rarity). These can be varied to control the learning difficulty. For example, if the peaks in $A C_{b}$ are wider, then more examples of $\mathrm{NC}$ get captured, making it difficult to achieve higher precision for reasonably high recall for C. Note that in type (II) model, the subclasses of type $N C_{a}$ have distribution peaks in both $A C O R R_{a}$ and $A N C_{a}$, that introduces a correlation between these attributes.

We generated multiple datasets with 1:20 ratio of $\mathrm{C}: \mathrm{NC}$ using the model (I) with 30,000 NC examples, and 1:10 ratio using model (II) with 20,000 NC examples. Some key results are presented in Tables 1.

The left table in part (A) shows that as the peak widths ${ }^{6} W C_{a}$ and $W C_{b}$ increase in model (I), the ability to remove false positives effectively is required, and PNrule outperforms AdaCost. The performance difference is less dependent on the the widths of $\mathrm{NC}$ peaks, implying that PNrule's approach of explicitly and collectively removing false positives is helping it and AdaCost fails to imitate this ability.

Right two tables of part (A) show similar results with the type (II) model of Figure 3. In particular, the top right table shows the effect of adding correlations. sc- 0 and sc- 1 both have identical parameters ${ }^{7}$, except that sc- 0 has no attributes of type $A C O R R_{a}$ or $A N C_{a}$ (i.e. $n_{A C O R R_{a}}=n_{A N C_{a}}=0$ ) and sc-1 has correlations between 3 pairs of $A C O R R_{a}$ and $A N C_{a}$ type attributes. The numbers $n_{A C O R R_{a}}+n_{A C_{b}}$ and $n_{A N C_{a}}+n_{A N C_{b}}$ is same for both datasets. It is difficult to discriminate between correlated parts of $C_{a}$ and $N C_{a}$ in sc-1,

\footnotetext{
${ }^{6}$ Compare widths to a range of 50 for all attributes. Other parameters for these datasets are $n_{A C O M_{a}}=3, p_{A C O M_{a}}=3, n_{A C_{b}}=4, p_{A C_{b}}=5, n_{A N C_{b}}=5$, and $p_{A N C_{b}}=5$.

${ }^{7} p_{C}=4, p_{N C}=8, W_{C}=0.2, W_{N C}=2$.
} 
thus $F_{1}$ value drops for all algorithms. But, $C_{a}$ can still be discriminated against $N C_{b}$ and parts of $N C_{a}$, provided an algorithm can collect and learn from only their relevant false positives (the explicit learning approach). PNrule has this ability. As the results indicate, AdaCost fails to impart this ability to IREP*-1 or IREP*-2. The lower right table of part (A) further corroborates our claim because as $p_{N C}$, the number of peaks over $A N C_{a}$ and $A N C_{b}$, increases in model (II), the false positives get scattered more, requiring an effect of explicit learning.

Part (B) of Table 1 justifies the use of AdaCost as the strongest boostingbased competitor of PNrule for the two difficult datasets from each model.

Finally, we demonstrate the effect of varying the class proportions on a moderately difficult dataset snc-6 from model (I) and the representative dataset sc-1 from model (II). The results in Table $1(\mathrm{C})$ indicate that PNrule is significantly better for rarer problems (as Cfrac $\downarrow$ ). Especially with correlations, PNrule seems to be better even for larger proportions of $\mathrm{C}$. This is interesting because the problem of separating correlated $\mathrm{C}$ and $\mathrm{NC}$ is difficult in general, and is made more difficult by the rarity. However, the ability to remove false positives collectively is required irrespective of the rarity, and on this count, PNrule outperforms AdaCost.

Due to lack of space, we have shown results on representative datasets, but similar results were observed for a wide variation of different parameters of the models.

\subsection{Results on Real and Benchmark Datasets}

We used the OHSU Medline document corpus of medical abstracts from years 1990 and 1991 [15]. Out of 805 topics having a population of $>0.2 \%$ (in around 148,000 total documents), we select 33 topics (least rare topic has about 5\% population). Some example topics are AIDS, Aging, Anoxia, Colon, Parkinson Disease, Placenta, etc. For each topic, after stemming and stop-word removal, we intersect the top 75 distinguishing words according to the mutual information and Z-number [2] metrics, to form the attribute set for its binary classification. According to the $95 \%$ confidence p-test, PNrule vs. AdaCost comparison is 326-4-0; i.e., PNrule outperforms AdaCost on 3 datasets, both are comparable on 26 , AdaCost is better on 4 , and on no dataset is IREP* better than the two ${ }^{8}$. This indicates that only in 4 cases, use of AdaCost is justifiable based on its high performance despite its low interpretability. On 29 cases, using more interpretable PNrule model is preferable.

We also did experiments on datasets from the UCI machine learning repository [16]. We form a binary problem for each class having $<35 \%$ proportion in the king-rook-king, dna, csb-smoke, led +17 , led, and letter datasets ${ }^{9}$. Out of 49 such problems, PNrule vs. AdaCost comparison is 7-23-18-1. This shows

\footnotetext{
${ }^{8}$ AdaCost is sometimes worse than its base learner because of our 1-way crossvalidation method of choosing the optimal number of iterations for AdaCost.

${ }^{9}$ king-rook-king is recognized as a tough problem for propositional learning, other datasets were chosen because they contain many rare classes
} 
Table 1. Results on synthetic models of Figure 3, comparing $F_{1}$ performance. Bold numbers indicate statistically better or comparable (95\% confidence ptest) algorithms. (A) Effect of varying model parameters. (B) Comparing various boosting algorithms for datasets snc-9 and sc-1. ABst: AdaBoost, RB1/2: RareBoost-1/2, SLIP: SLIPPER [6], ACst: AdaCost. CSB1 and CSB2 are from [7]. Refer to [9] for RB-1 and RB-2. (C) Results for varying proportion of class $\mathrm{C}$ in the training set (Cfrac) for datasets snc-6 and sc-1

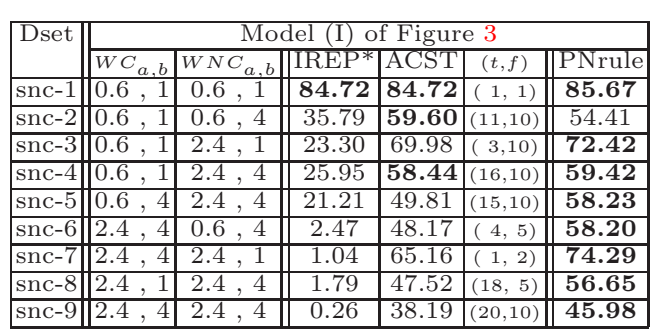

(A)

\begin{tabular}{|c|c|c|c|c|}
\hline \multirow[t]{2}{*}{ Dset } & \multicolumn{4}{|c|}{ Model (II) of Figure 3} \\
\hline & IREP* & $\mathrm{ACST}$ & $(t, f)$ & PNrule \\
\hline $\mathrm{sc}-0$ & 85.45 & 89.08 & $(3,10)$ & 88.45 \\
\hline sc-1 & 41.65 & 41.65 & $(1,1)$ & 59.03 \\
\hline$p_{N C}$ & & Dset & sc-1 & \\
\hline 2 & 67.75 & 72.00 & $(12,5)$ & 73.33 \\
\hline 4 & 32.11 & 60.96 & $(46,5)$ & 57.10 \\
\hline 8 & 41.65 & 41.65 & $(1,1)$ & 59.03 \\
\hline 12 & 41.17 & 50.13 & $(47,5)$ & 56.59 \\
\hline 20 & 3.90 & 28.57 & $(21,5)$ & 51.21 \\
\hline
\end{tabular}

Dset |ABst|RB-1||SLIP $\mid$ RB-2||CSB1|CSB2| ACst

\begin{tabular}{|l|l|l|l|l|l|l|l|l|l|l|l|l|}
\hline snc-9 & 26.60 & 31.78 & 16.65 & 32.96 & 30.71 & 24.91 & 45.98 \\
\hline
\end{tabular}

\begin{tabular}{|l|l|l|l|l|l|l|l|l|l|l|l|}
\hline \hline sc- 1 & 23.43 & 28.39 & 20.23 & 34.15 & 36.75 & 37.00 & 41.65 \\
\hline
\end{tabular}

(B)

\begin{tabular}{|c|c||c|c||c|}
\hline \multirow{2}{*}{ Cfrac } & \multicolumn{4}{|c|}{ Dset: snc-6 } \\
\cline { 2 - 5 } & IREP* & ACST & $(t, f)$ & PNrule \\
\hline $2.44 \%$ & 0.00 & 7.43 & $(3,10)$ & $\mathbf{1 8 . 5 3}$ \\
\hline $4.76 \%$ & 2.47 & 48.17 & $(4,5)$ & $\mathbf{5 8 . 2 0}$ \\
\hline $9.09 \%$ & 8.79 & 53.37 & $(14,5)$ & $\mathbf{6 0 . 3 0}$ \\
\hline $16.67 \%$ & 60.62 & $\mathbf{6 8 . 2 1}$ & $(9,5)$ & 66.12 \\
\hline $28.57 \%$ & 47.10 & $\mathbf{7 6 . 6 0}$ & $(17,2)$ & 71.82 \\
\hline $50.00 \%$ & 74.94 & $\mathbf{8 1 . 4 5}$ & $(4,1)$ & 69.11 \\
\hline
\end{tabular}

\begin{tabular}{|c|c||c|c||c|}
\hline Cfrac & \multicolumn{4}{|c|}{ Dset: sc-1 } \\
\cline { 2 - 5 } & IREP* & ACST & $(t, f)$ & PNrule \\
\hline $2.44 \%$ & 0.00 & 0.76 & $(1,5)$ & $\mathbf{1 3 . 9 7}$ \\
\hline $4.76 \%$ & 1.55 & 15.38 & $(31,10)$ & $\mathbf{1 9 . 1 4}$ \\
\hline $9.09 \%$ & 41.65 & 41.65 & $(1,1)$ & $\mathbf{5 9 . 0 3}$ \\
\hline $16.67 \%$ & 3.22 & 45.80 & $(17,5)$ & $\mathbf{7 9 . 7 3}$ \\
\hline $28.57 \%$ & 23.09 & 59.89 & $(12,2)$ & $\mathbf{8 0 . 4 8}$ \\
\hline $50.00 \%$ & 65.01 & $\mathbf{7 8 . 4 4}$ & $(1,2)$ & $\mathbf{7 8 . 5 3}$ \\
\hline
\end{tabular}

(C)

existence of 31 of 49 scenarios, where use of AdaCost is not justifiable. On the 17 datasets formed from the king-rook-king problem, the comparison is 6-7-4-0; i.e., on 13 of 17 datasets, PNrule is either comparable or better than AdaCost.

\section{Concluding Remarks}

The problem of achieving better balance between recall and precision while predicting a rare event class is challenging. In this context, we compare PNrule, a strong non-meta technique of two-phase rule induction to AdaCost, a strong cost-sensitive boosting methodology that was shown to outperform other boosting algorithms from the recall-precision perspective. The non-meta techniques have an advantage of being interpretable. We argue via detailed qualitative analysis that boosting lacks a crucial ability of PNrule to collect only the relevant 
false positives and explicitly learn rules to exclude them. The arguments are supported using various synthetic and real-world datasets. PNrule is especially better for rarer classes, and when there is a strong correlation among the distinguishing rules of the rare class and the non-rare class. As an extension of the observations and arguments of this paper, one can investigate two aspects w.r.t. rare class problems: a. Does the boosting performance depend crucially on the base learner?, b. Can one improve boosting by using an explicit learning method of PNrule at its base learner?. Our work on these issues appears at the ACM KDD'2002 conference.

\section{Acknowledgments}

The contribution to this work by Prof. Vipin Kumar was supported in part by the Army High Performance Computing Research Center cooperative agreement number DAAD19-01-2-0014, the content of which does not necessarily reflect the position or the policy of the government, and no official endorsement should be inferred.

\section{References}

1. Holte, R. C., Japkowicz, N., Ling, C. X., Matwin, S.: (eds.) Learning from imbalanced data sets workshop. Technical Report WS-00-05, AAAI Press (2000) 237

2. Agarwal, R. C., Joshi, M. V.: PNrule: A new framework for learning classifier models in data mining (A case-study in network intrusion detection). In: Proceedings of First SIAM Conference on Data Mining, Chicago (2001) 237, 239, 240, 244, 246

3. Joshi, M. V., Agarwal, R. C., Kumar, V.: Mining needles in a haystack: Classifying rare classes via two-phase rule induction. In: Proc. of ACM SIGMOD Conference, Santa Barbara, CA (2001) 91-102 237, 238, 239, 240, 245

4. van Rijsbergen, C. J.: Information Retrieval. Butterworths, London (1979) 238, 243

5. Schapire, R., Singer, Y.: Improved boosting algorithms using confidence-rated predictions. Machine Learning 37 (1999) 297-336 238, 239, 242

6. Cohen, W., Singer, Y.: A simple, fast, and effective rule learner. In: Proc. of Annual Conference of American Association for Artificial Intelligence. (1999) 335-342 238, 239, 247

7. Ting, K. M.: A comparative study of cost-sensitive boosting algorithms. In: Proc. of 17th Intl. Conf. on Machine Learning. (2000) 983-990 238, 239, 247

8. Fan, W., Stolfo, S. J., Zhang, J., Chan, P. K.: AdaCost: Misclassification costsensitive boosting. In: Proc. of 6th Intl. Conf. on Machine Learning (ICML). (1999) 238, 239

9. Joshi, M. V., Kumar, V., Agarwal, R. C.: Evaluating boosting algorithms to classify rare classes: Comparison and improvements. In: Proc. of The First IEEE International Conference on Data Mining (ICDM), San Jose, CA (2001) 238, 239, 247 
10. Cohen, W. W.: Fast effective rule induction. In: Proc. of Twelfth International Conference on Machine Learning, Lake Tahoe, California (1995) 238, 240, 243, 244

11. Quinlan, J. R.: C4.5: Programs for Machine Learning. Morgan Kaufmann (1993) 240

12. Vere, S. A.: Multilevel counterfactuals for generalizations of relational concepts and productions. Artificial Intelligence 14 (1980) 139-164 240

13. Compton, P., Jansen, R.: A philosophical basis for knowledge aquisition. Knowledge Acquisition 2 (1990) 241-257 240

14. Yang, Y., Liu, X.: A re-examination of text categorization methods. In: 22nd Annual Intl. Conf. on Information Retrieval (SIGIR). (1999) 42-49 244

15. Hersh, W., Buckley, C., Leone, T., Hickam, D.: OHSUMED: An interactive retrieval evaluation and new large test collection for research. In: In Proceedings of the 17th Annual ACM SIGIR Conference. (1994) 192-201 246

16. Blake, C., Merz, C.: UCI repository of machine learning databases (1998) http://www.ics.uci.edu/ mlearn/MLRepository.html. 246 